\title{
Modeling the transient flow of undercooled glass-forming liquids
}

\author{
Marios D. Demetriou ${ }^{\mathrm{a})}$ and William L. Johnson \\ Keck Laboratory of Engineering Materials, California Institute of Technology, Pasadena, California 91125
}

(Received 23 June 2003; accepted 8 December 2003)

\begin{abstract}
In a recent experimental study on flow behavior of Vitreloy-1 $\left(\mathrm{Zr}_{41.25} \mathrm{Ti}_{13.75} \mathrm{Cu}_{12.5} \mathrm{Ni}_{10} \mathrm{Be}_{22.5}\right)$, three distinct modes of flow are suggested: Newtonian, non-Newtonian, and localized flow. In a subsequent study, the experimental flow data is utilized in a self-consistent manner to develop a rate equation to govern local free volume production. In the present study the production-rate equation is transformed into a transport equation that can be coupled with momentum and energy transport via viscosity to formulate a model capable to govern the flow of undercooled glass forming liquids. The model is implemented to study the flow behavior of undercooled Vitreloy-1 melt. For a temperature of $700 \mathrm{~K}$ and shear loading of $1.0 \mathrm{MPa}$, the model predicts that the flow profile gradually stabilizes to its Newtonian limit while the liquid is maintained in structural and thermal equilibrium. For the conditions of $675 \mathrm{~K}$ and $100 \mathrm{MPa}$, the model predicts that the flow profile departs from its Newtonian limit and gradually stabilizes to a non-Newtonian limit. The non-Newtonian profile is evaluated independently by considering structurally quasistatic conditions, which yield the shear-rate dependency of flow. For the conditions of $650 \mathrm{~K}$ and $2.0 \mathrm{GPa}$, the model predicts that the flow continuously localizes and ultimately accelerates unconstrained, while the system is driven out of structural and thermal equilibration towards an unstable state associated with free volume generation, viscosity degradation, and temperature rise. The computed temperature and shear rate evolutions for the three distinct flow modes are superimposed on a temperature-shear rate diagram and appear to computationally reproduce the experimental flow map. The system's structural state that appears to dictate flow behavior is quantified by a dimensionless number, which results from a time scale analysis of the free volume production equation. (C) 2004 American
\end{abstract} Institute of Physics. [DOI: 10.1063/1.1645669]

\section{INTRODUCTION}

Within the theoretical mechanics community, the study of shear band formation has been a topic of ongoing interest. It has been conventionally perceived that shear bands form upon deformation close to or below glass transition, and thus most of the early work was devoted to shear band formation in metallic glasses. Production experiments by Liquidmetal Technologies on injection casting of Vitreloy-1 $\left(\mathrm{Zr}_{41.25} \mathrm{Ti}_{13.75} \mathrm{Cu}_{12.5} \mathrm{Ni}_{10} \mathrm{Be}_{22.5}\right)$, however, indicate that shear bands may also form upon deformation above glass transition in the deeply undercooled liquid region. Figure 1 shows an angular corner of a Vitreloy-1 cast part. During mold filling of this part the melt was maintained above glass transition temperature, which implies that material deformation occurred in the undercooled liquid state. A zoom in the angular corner reveals the existence of multiple shear bands. It appears that around the angular corner where the molten melt had to undergo extremely high shearing rates (sharp change in speed and direction), the flow became localized and ultimately evolved into serrated flow generating multiple shear bands perpendicular to the flow direction.

Recently Lu et al. ${ }^{1}$ carried out an experimental study on the flow behavior of Vitreloy-1. An experimental flow diagram is produced, which is presented in Fig. 2. The diagram

\footnotetext{
a) Author to whom correspondence should be addressed; electronic mail: marios@caltech.edu
}

outlines the boundaries between three distinct flow modes: Newtonian, non-Newtonian, and shear localization. Newtonian flow is realized at high temperatures and low shearing rates and is maintained independent of shear rate. NonNewtonian flow is realized at moderate temperatures and shearing rates and is essentially shear-rate dependent, however the dependency arises as a consequence of structural stabilization in the liquid which contributes to the flow remaining stable. Shear localization is realized at low temperatures and high shearing rates. Under those conditions the flow becomes extremely sensitive to shear rate and as a consequence it is driven out of stability. The plot suggests that the range of temperature and shearing rate associated with shear localization which contributes to shear banding is not bounded by glass transition but carries onto the deeply undercooled liquid region, hence providing further evidence for shear banding in undercooled liquids.

Shear localization in undercooled liquids and glasses is in fact caused by a local reduction in viscosity in the vicinity of a stress concentration, and evolves to dramatically increase and confine the deformation until it approaches a local instability, which is termed shear band. The decrease in viscosity during deformation is a consequence of increasing free volume and thus decreasing density, which severely reduces the material's resistance to deformation. Early work ${ }^{2-5}$ identified two potential origins of shear localization: thermal expansion of molar volume induced by adiabatic heating which causes the free volume to increase, and structural disordering 


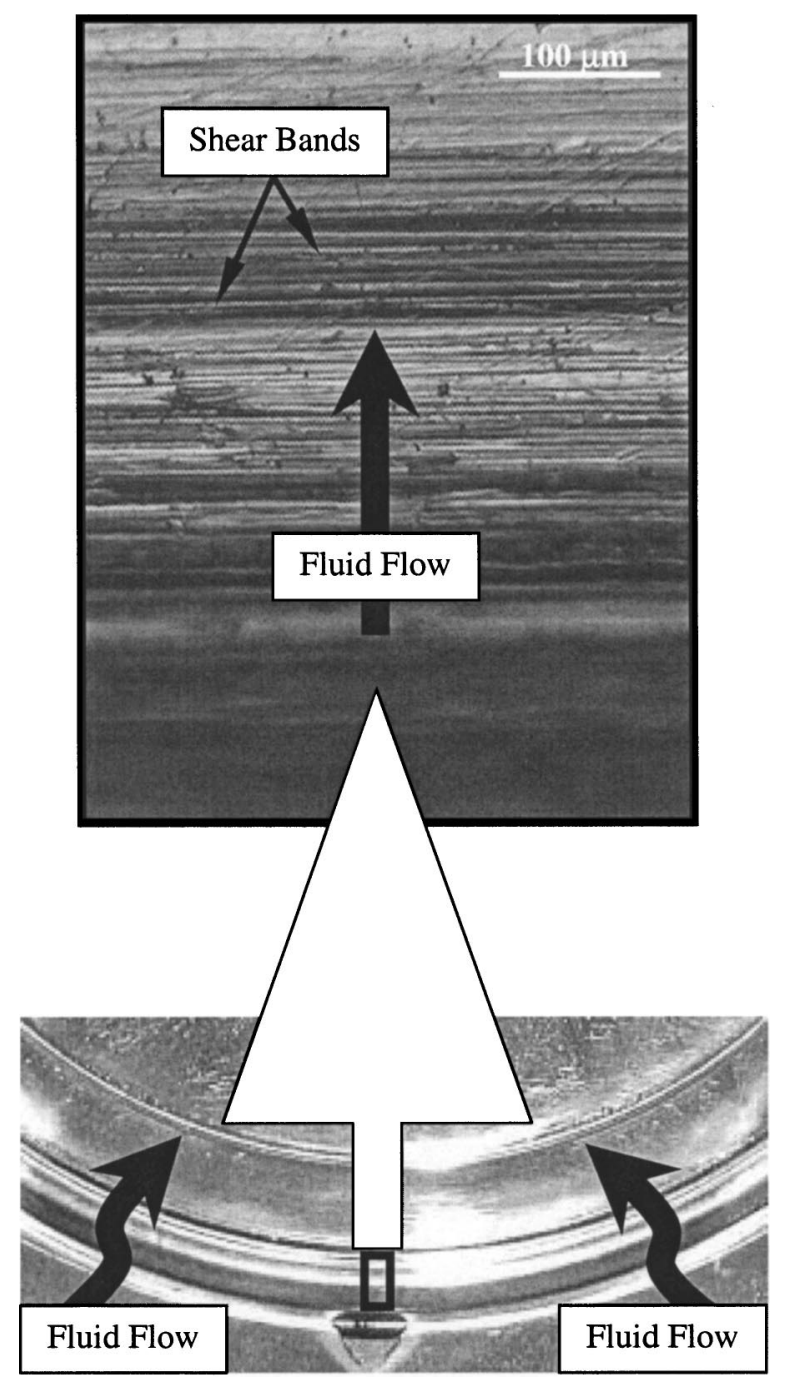

FIG. 1. A corner from a Vitreloy-1 cast part produced by Liquidmetal Technologies. Around the angular corner the melt had to undergo excessively high shearing rates. Consequently the flow became localized and developed serrations generating multiple shear bands across the flow direction.

in the liquid induced by shear which contributes to free volume creation. Although significant heating is generated during shear localization, recent evidence ${ }^{6-9}$ indicates that adiabatic heating is not the prime origin, as localization appears to be triggered by structural disordering. A model based on free volume production dictated by both adiabatic heating and structural disordering would form an appropriate basis for understanding the mechanisms triggering shear localization.

More recently Johnson et al. ${ }^{10}$ utilized the experimental flow data of Lu et al. ${ }^{1}$ in a self-consistent manner to formulate a simple constitutive flow law applicable to glass forming metallic liquids undergoing steady flow. The model, which is essentially a simple treatment of free volume production and annihilation during flow, is based on the assumption that shearing results in the creation of free volume such as a relationship between free volume and shear rate is obtained by a self-consistency argument. The flow law was implemented to obtain the viscosity dependence on shear rate under structurally steady-state conditions. More recently

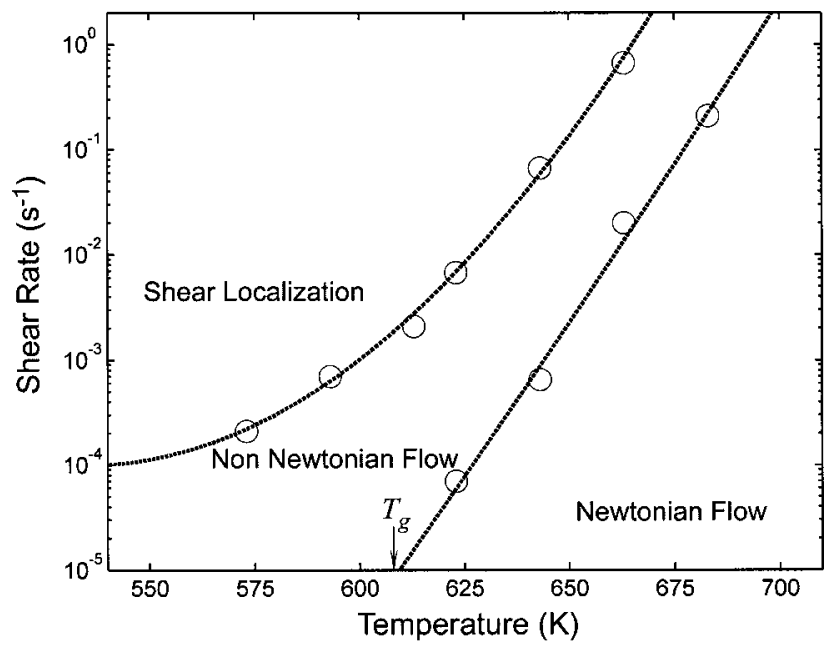

FIG. 2. Results from Vitreloy-1 flow experiments carried out by Lu et al. (Ref. 1). The boundaries for the transition from Newtonian to nonNewtonian flow and from non-Newtonian to localized flow are shown. Each flow mode is associated with a range of shearing rates and temperatures, which extends above glass transition $T_{g}$ into the undercooled liquid region.

Bossuyt et al. ${ }^{11}$ extended the model to include transient effects and simulated the stress-strain response during deformation.

In the present study, the rate equation governing free volume production developed by Johnson et al. ${ }^{10}$ is transformed into a transport equation, which under the assumption of incompressible flow it can be regarded as a conservation equation for free volume. This equation is coupled with the conservations of momentum and energy via viscosity to form a complete transport model applicable to bulk glass-forming liquids (such as those of the $\mathrm{Zr}-\mathrm{Ti}-\mathrm{Ni}-\mathrm{Cu}-\mathrm{Be}$ Vitreloy alloy family) undergoing deformation. The developed transport model is capable of computing transient free volume distributions as dictated by adiabatic heating and structural disordering, and hence simulating the transient flow evolution. The model is implemented to simulate the transient flow of Vitreloy-1 under asymmetric shear loading, as such loading could trigger shear localization. The simplest flow model that involves asymmetric shear is the one dimensional (1D) pressure driven flow, or "Poiseuille" flow, in which the shear loading is imposed at the channel wall by the applied pressure gradient. The aim of this study is to assess the potential of the model in simulating the flow characteristics of the three flow modes illustrated on the experimental flow map in Fig. 2.

\section{FREE VOLUME TRANSPORT MODEL}

Assuming the validity of Vogel-Fulcher-Tamann equation far from the Newtonian limit, the viscosity dependence on free volume may be expressed in the form ${ }^{10}$

$$
\eta(\nu)=\eta_{\infty} \exp \left(\frac{\Phi \alpha \nu_{o} T_{o}}{\nu-\nu_{o}}\right),
$$

where $\nu$ is the molar volume, $\nu_{o}$ is the molar volume at the Vogel-Fulcher-Tammann temperature $T_{o}, \nu-\nu_{o}$ is the free volume, $\Phi$ is the liquid "fragility" index, and $\alpha$ is the volume thermal expansion coefficient (assumed temperature in- 
dependent). The infinite-shear-rate limit of viscosity can be approximated as $\eta_{\infty}=h_{p} N_{a} / \nu_{e}$, where $h_{p}$ is Planck's constant, $N_{a}$ is Avogadro's number, and $\nu_{e}$ is the molar volume of a relaxed liquid, i.e., the molar volume of a liquid under structural equilibration dictated only by temperature. Its temperature dependence is given by

$$
\nu_{e}=\nu_{o}\left[1+\alpha\left(T-T_{o}\right)\right] .
$$

It is worth noting that the limit of viscosity at the equilibrium free volume, $\eta\left(\nu \rightarrow \nu_{e}\right)$, is the Newtonian limit $\eta_{0}$ given by

$$
\eta_{0}(T)=\eta_{\infty} \exp \left(\frac{\Phi T_{o}}{T-T_{o}}\right) .
$$

For Vitreloy-1, the parameters above take the following values: $T_{o}=412 \mathrm{~K} ; \Phi=19.8 ; \nu_{o}=9.766 \times 10^{-6} \mathrm{~m}^{3} / \mathrm{mole} ;$ and $\alpha=5.32 \times 10^{-5} \mathrm{~K}^{-1}$.

Shearing under a finite shear rate $\dot{\gamma}$ results in liquid disordering, which can be quantified by creation of excess volume proportional to the total shear strain as $\dot{\nu}_{\text {cre }}=R \dot{\gamma}$, where the parameter $R$ is the free volume creation coefficient and describes the molar volume produced by a unit plastic shear strain. For Vitreloy-1, this parameter was determined by optimization in the study of Johnson et al. ${ }^{10}$ to be $R=0.19 \nu_{o}$. The excess free volume created by shear will tend to be annihilated by relaxation of the total free volume towards equilibrium. ${ }^{12}$ The rate of relaxation can be related to the relaxation time for viscosity toward its equilibrium value as $\dot{\nu}_{\text {rel }}=\left(\nu-\nu_{e}\right) / \tau_{\nu, \text { rel }}$. In the simplest model for liquid viscoelastic behavior the configurational relaxation time is taken as $\tau_{\nu \text {,rel }}=\eta(\nu) / G$, where $G$ is the isoconfigurational liquid shear modulus in the high-frequency limit and can be approximated by the static shear modulus of low temperature glass (33 GPa for Vitreloy-1). The local time-rate of change of free volume can then be expressed as

$$
\frac{d \nu}{d t}=\dot{\nu}_{\mathrm{cre}}-\dot{\nu}_{\mathrm{rel}}=R \dot{\gamma}-\frac{\nu-\nu_{e}}{\eta / G},
$$

Assuming continuum in the vicinity of localization and introducing a transport field that can accommodate free volume convection and diffusion, a conservation equation governing free volume transport may be derived as

$$
\frac{\partial \nu}{\partial t}+\mathbf{u} \cdot \boldsymbol{\nabla} \nu=\boldsymbol{\nabla} \cdot(D \boldsymbol{\nabla} \nu)+R \dot{\gamma}-\frac{\nu-\nu_{e}}{\eta / G},
$$

where $\mathbf{u}$ is the velocity field. In such transport field, the shear rate tensor is given as $\dot{\gamma}=\boldsymbol{\nabla} \mathbf{u}+(\boldsymbol{\nabla u})^{\dagger}$, and its magnitude as $\dot{\gamma}=\sqrt{1 / 2(\dot{\gamma} \cdot \dot{\gamma})}$. The free-volume diffusivity $D$ can be taken as equivalent to the liquid self-diffusion coefficient, since free volume diffusion essentially implies atomic rearrangement. However since diffusion coefficients characterizing such liquids are extremely small, the diffusive divergence in Eq. (5) turns out to be insignificant.

Practically, Eq. (5) could be coupled to the transport equations that govern conservation of mass, momentum and energy given, respectively, below

$$
\boldsymbol{\nabla} \cdot \mathbf{u}=0,
$$

$$
\begin{aligned}
& \rho\left(\frac{\partial \mathbf{u}}{\partial t}+\mathbf{u} \cdot \boldsymbol{\nabla} \mathbf{u}\right)=\boldsymbol{\nabla} \cdot(\eta \dot{\gamma})-\boldsymbol{\nabla} p, \\
& \rho c_{p}\left(\frac{\partial T}{\partial t}+\mathbf{u} \cdot \boldsymbol{\nabla} T\right)=\boldsymbol{\nabla} \cdot(k \boldsymbol{\nabla} T)+\eta(\dot{\gamma}: \dot{\gamma}),
\end{aligned}
$$

where $p$ is pressure, $\rho$ is the density, $c_{p}$ is the specific heat, and $k$ is the thermal conductivity. Equations (5)-(8) represent a set of transport equations coupled through viscosity, shear rate, and temperature. This set of equations could adequately simulate the transient flow evolution of bulk glassforming liquids under deformation. Moreover provided that a suitable adaptive mesh is employed, the evolution of shear instabilities, which arise as a consequence of the highly nonlinear dependence of viscosity on free volume [Eq. (1)], could be accommodated. However as this model is based on the assumption of continuum in the vicinity of localization, it would fail to simulate a shear band as it stands physically, i.e., having finite thickness and being associated with a finite shear rate, but would tend to model a shear band in a more mathematical sense, i.e., as a mathematical instability characterized by zero thickness and associated with an infinite shear rate.

In the context of this study, the system of equations is simplified and solved for the case of 1D pressure-driven flow (Poiseuille flow) in a channel of thickness $2 L$. Although overly simplified, such flow conditions in their unstable limit would replicate the instabilities associated with shear band initiation during a single serration. From this perspective, this study constitutes a valuable means for analyzing the dynamics of shear banding in bulk liquids. The transport equations simplified for Poiseuille flow are

$$
\begin{aligned}
& \rho \frac{\partial u}{\partial t}=\frac{\partial}{\partial x}\left[\eta(\nu) \frac{\partial u}{\partial x}\right]+p^{\prime}, \\
& \frac{\partial \nu}{\partial t}=R\left|\frac{\partial u}{\partial x}\right|-\frac{\nu-\nu_{e}}{\eta(\nu) / G}, \\
& \rho c_{p} \frac{\partial T}{\partial t}=\frac{\partial}{\partial x}\left[k \frac{\partial T}{\partial x}\right]+\eta(\nu)\left(\frac{\partial u}{\partial x}\right)^{2},
\end{aligned}
$$

where $p^{\prime}$ is the applied pressure gradient, $\rho=M / \nu$ is the density $(M=0.0603 \mathrm{~kg} / \mathrm{mole}$ for Vitreloy), while the shear rate is simply $\dot{\gamma}=|\partial u / \partial x|$ The specific heat is given by $c_{p}$ $=(1 / M)\left(24.9+7.5 \times 10^{-3} T+8.17 \times 10^{6} / T^{2}\right) \mathrm{J} / \mathrm{kg} \mathrm{K},{ }^{13}$ and the thermal conductivity by $k=0.0163 T+0.8407 \mathrm{~W} / \mathrm{m} \mathrm{K}^{14}$ Free volume diffusion is neglected.

\section{ANALYSIS}

Momentum transport, Eq. (9), is characterized by a relaxation time scale $\tau_{u \text {,rel }} \sim \rho L^{2} / \eta$. This relaxation time characterizes the time required for the flow profile to develop its Newtonian distribution, which for such 1D flow is parabolic. However if substantial deformation is sustained for $t$ $\gg \tau_{u \text {,rel }}$, further evolution of the profile could be realized. The post-Newtonian flow evolution can be taken to progress quasistatically, i.e., $\rho \partial u / \partial t=0$, which implies that the shear loading, defined as $\sigma \equiv \eta(t) \dot{\gamma}(t)=p^{\prime} x$, would remain constant in time. This suggests that the viscosity degrades at the 


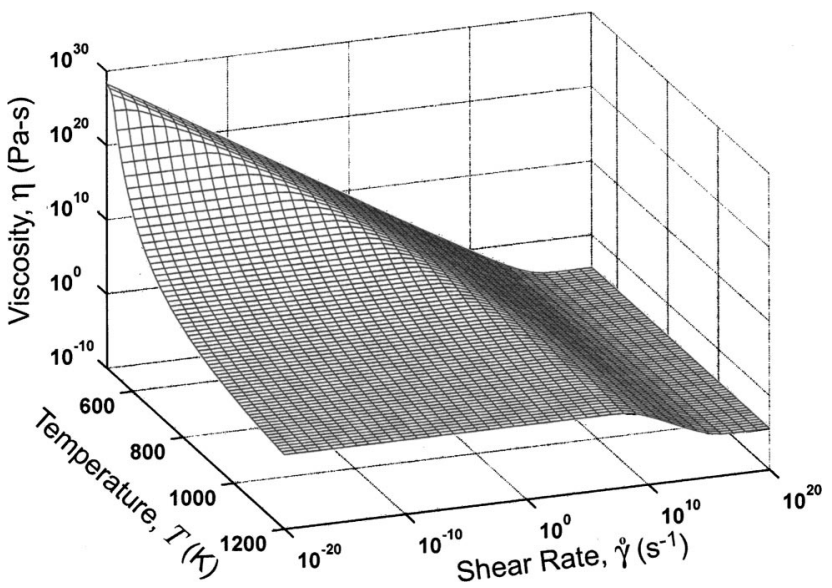

FIG. 3. Viscosity dependence on shear rate and temperature for Vitreloy-1 computed from Eq. (13). At low shearing rates and high temperatures, the viscosity remains near the Newtonian limit governed by the Vogel-FulcherTamann viscosity law. At higher shearing rates and lower temperatures, the viscosity dependence on shear rate becomes more pronounced as the free volume produced by shearing has a degrading effect on viscosity.

same rate as the shear rate evolves such that their product $\sigma$ is maintained constant in time. Since nonlinear elasticity theory is not incorporated in the model, the shear loadings considered in this study were taken not to exceed the material shear strength $(\sim 2.0 \mathrm{GPa}$ for Vitreloy-1) so as to warrant the validity of the model.

Free volume transport, Eq. (10), is characterized by a relaxation time scale $\tau_{\nu, r e l} \sim \eta / G$. This time scale characterizes the time required for the liquid to structurally equilibrate. Under non-Newtonian flow conditions, the induced shear produces an amount of free volume, which on one hand is large enough to cause substantial viscosity degradation, while on the other, is small enough to be annihilated. Consequently the flow becomes shear-rate dependent yet it remains stable, while the flow profile relaxes to its nonNewtonian limit. The shear rate dependence on flow can be obtained by setting Eq. (4) to zero, i.e., equating local free volume production to free volume annihilation:

$$
R \dot{\gamma}=\frac{\nu-\nu_{e}}{\eta / G} .
$$

Substituting Eqs. (1) and (2) into Eq. (12), a self-consistency condition for the viscosity at a finite shear rate can be obtained as follows: ${ }^{10}$

$$
\eta(T, \dot{\gamma})=\eta_{\infty} \exp \left[\frac{\Phi \alpha \nu_{o} T_{o}}{\nu_{o} \alpha\left(T-T_{o}\right)+(R / G) \dot{\gamma} \eta(T, \dot{\gamma})}\right] .
$$

Equation (13) is essentially an implicit formulation for the viscosity dependence on temperature and shear rate under structurally nonequilibrium steady-state conditions. Equation (13) can be solved numerically over a wide range of temperatures and shear rates. The solution for Vitreloy- 1 in the temperature range of $550-1200 \mathrm{~K}$ and shear rate of $10^{-20}-10^{20} \mathrm{~s}^{-1}$ obtained using the Newton-Raphson method is shown in Fig. 3. At sufficiently low shearing rates the viscosity remains near its Newtonian limit $\eta_{0}$ given by Eq. (3), while at excessively high shearing rates the viscosity

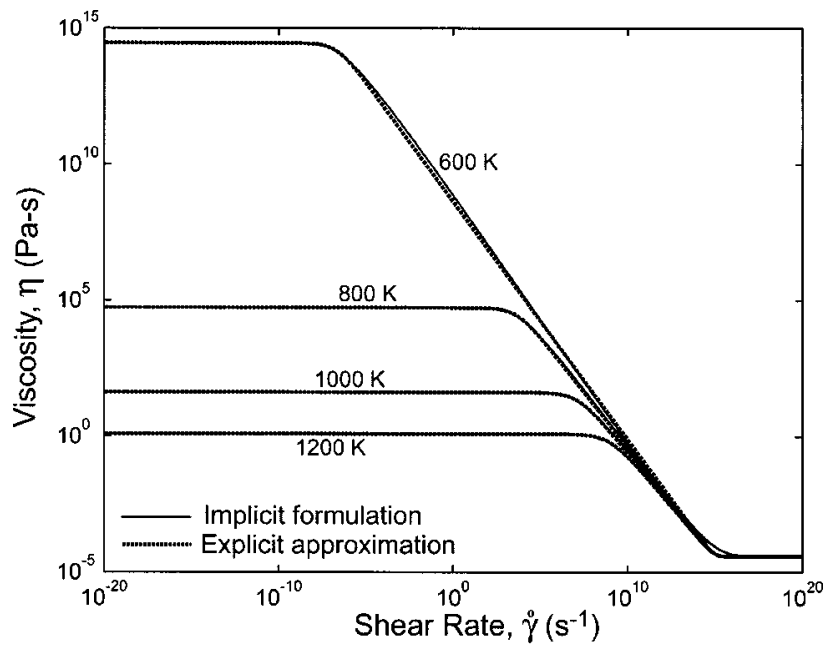

FIG. 4. Viscosity dependence on shear rate at different temperatures for Vitreloy-1 computed from Eqs. (13) and (14). The explicit approximation accurately fits the implicit formulation for the shear rate dependent viscosity over a wide range of shear rate and temperature.

approaches its infinite-shear-rate limit $\eta_{\infty}$. At any given temperature a range of shear rate exists for which the decaying dependence of viscosity on shear rate becomes evident. This decaying range, which varies with temperature, is termed shear-rate "stretching" and is attributed to free volume production, which has a degrading effect on viscosity. It should be noted, however, that at very high shearing rates and very low temperatures the solution is not justifiable, as the time scales characterizing free volume annihilation become excessively large and the assumption of structurally quasistatic flow breaks down.

In the interest of providing a concise analytical expression for non-Newtonian steady-state viscosity, an analytic fit to the numerical solution was carried out. The fitting was accomplished by considering the limiting cases of $\eta(\dot{\gamma}$ $\rightarrow 0)=\eta_{0}$ and $\eta(\dot{\gamma} \rightarrow \infty)=\eta_{\infty}$, and by realizing that a characteristic shear rate is given by $G / \eta_{\infty}$. Moreover, the temperature variation of the shear-rate "stretching" of viscosity in the non-Newtonian regime was considered. Physically, the "stretching" behavior is attributed to the existence of a spectral distribution of relaxation events, whose average and variance vary with temperature. In order to accommodate the temperature variation of the relaxation distribution variance, a "stretching" shear-rate exponent $\beta(T)$ was introduced in the approximation. The fitting formulation is given by

$$
\eta(T, \dot{\gamma})=\frac{\eta_{0}}{1+\left(\eta_{0} / \eta_{\infty}-1\right) \tanh \left\{\left[\dot{\gamma} /\left(G / \eta_{\infty}\right)\right]^{\beta(T)}\right\}} .
$$

The temperature dependence of the "stretching" exponent, which was the only fitting parameter in the approximation, was approximated by the following polynomial function:

$$
\beta(T)=2.5824 \times 10^{-7} T^{2}-6.5896 \times 10^{-4} T+1.1755 .
$$

As shown in Fig. 4, the explicit approximation given by Eq. (14) accurately fits the implicit formulation given by Eq. (13) over a wide range of temperature and shear rate. For shear rates of physical interest $\left(\dot{\gamma}<10^{10} \mathrm{~s}^{-1}\right)$ Eq. (14) can be simplified considerably as follows: 


$$
\eta(T, \dot{\gamma})=\frac{\eta_{0}}{1+\left(\eta_{0} / \eta_{\infty}\right)\left[\dot{\gamma} /\left(G / \eta_{\infty}\right)\right]^{\beta(T)}} .
$$

The shear rate dependency formulation given in Eq. (15) exhibits functional similarity to empirical formulations employed in rheological studies for the characterization of nonNewtonian fluids. In modeling non-Newtonian flows, structurally quasistatic conditions can be assumed such as Eq. (5) is rendered redundant and Eq. (15) can be used in conjunction with the momentum equation to accurately predict the flow evolution.

Energy transport, Eq. (11), is characterized by a relaxation time scale $\tau_{T, \text { rel }} \sim \rho c_{p} L^{2} / k$. This time scale characterizes the time required for the system to attain thermal equilibration. The thermal relaxation time is excessively large when compared to $\tau_{u \text {,rel }}$, though when small characteristic lengths are considered, as in the case of serrated flow where the characteristic length is the shear band spacing, this time scale could become comparable to $\tau_{\nu, r e l}$. In the current study, however, deformation is taken to occur locally in the bulk liquid such that the characteristic length is essentially the liquid dimension, which yields $\tau_{T, \text { rel }} \gg \tau_{\nu, \text { rel }}$. This implies that Eq. (11) would be unconditionally unstable in the time frame of deformation, as thermal relaxation would not be possible. The continuous rise in local temperature during deformation as a consequence of adiabatic heating causes local thermal expansion of the equilibrium molar volume, which increases free volume and could further exacerbate localization. The rate of local thermal expansion of the equilibrium molar volume, which translates into a rate of increase of free volume, can be approximated by $d \nu_{e} / d t$ $=\alpha \nu_{0} \eta \dot{\gamma}^{2} / \rho c_{p}$. As a matter of fact, in flows of less undercooled (higher temperature) liquids that remain structurally equilibrated, shear localization could be realized as a sole consequence of thermal expansion. Shear bands evolving from such thermally induced localization are termed "adiabatic."

\section{SIMULATION RESULTS}

Initially the melt is taken to be hydrodynamically, thermally, and structurally at equilibrium, i.e., it is considered to be at rest, at a uniform undercooling temperature $T_{i}$ and equilibrium free volume $\nu_{e}\left(T_{i}\right)$. At the channel boundaries, the no-slip condition is applied for velocity and vanishing Neumann (adiabatic) conditions are implemented for both temperature and free volume. The channel half thickness, which represents the bulk liquid, is taken to be $L=1 \mathrm{~m}$. The scientific package FEMLAB was employed to numerically integrate the transport equations by means of finite elements. All simulations were performed using a nonuniform mesh consisting of 67 linear Lagrange elements having a boundary element size of $10^{-6} \mathrm{~m}$ and a mesh growth rate 1.2 in order to accommodate the evolution of localization at the boundary. In order to evade numerical instabilities, artificial diffusion along the system characteristics was introduced.

\section{A. Newtonian flow}

To investigate the Newtonian flow regime, the initial temperature is taken to be $700 \mathrm{~K}$ and the pressure gradient

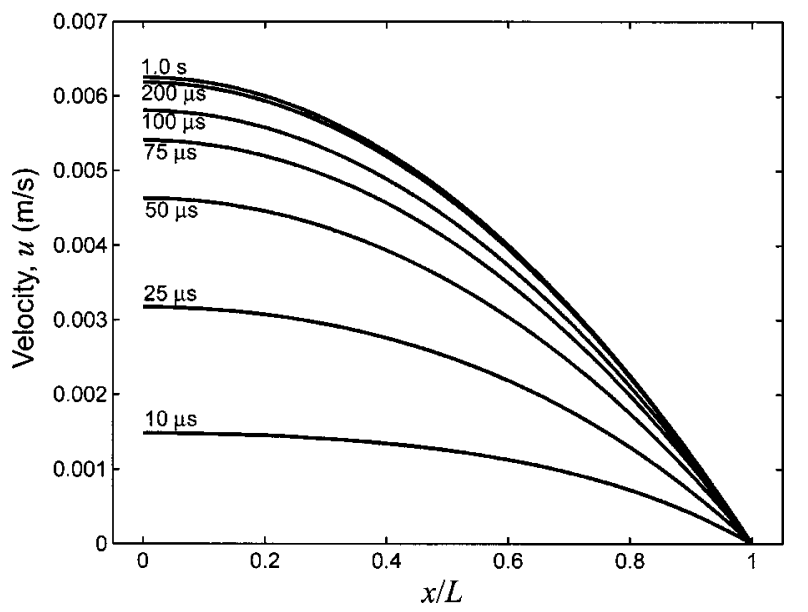

FIG. 5. Evolution of the velocity profile under $700 \mathrm{~K}$ and $1.0 \mathrm{MPa}$. The velocity has fully relaxed to its Newtonian profile and appears stable at this limit over extended periods of times.

1.0 $\mathrm{MPa} / \mathrm{m}$, which corresponds to a shear loading of 1.0 $\mathrm{MPa}$. The Newtonian viscosity at $700 \mathrm{~K}$ is $\sim 8 \times 10^{7} \mathrm{Pas}$. Newtonian hydrodynamics dictate that under these conditions the system relaxes at $\sim 75 \mu$ s to a parabolic profile characterized by $\sim 6.25 \mathrm{~mm} / \mathrm{s}$ maximum velocity at the center line and $\sim 1.25 \times 10^{-2} \mathrm{~s}^{-1}$ maximum shear rate at the wall.

The transient velocity distribution predicted by the model under these conditions is shown in Fig. 5. Within 200 $\mu$ s the velocity appears to have fully relaxed to its Newtonian profile. Moreover the flow appears stable at this limit over extended periods of times, as the distribution calculated at $1.0 \mathrm{~s}$ shows only a slight deviation. This deviation is attributed to insignificant amount of free volume generated at the wall during the period of $1.0 \mathrm{~s}$ that caused a slight reduction in the wall viscosity. Therefore since the liquid is maintained in structural and thermal equilibrium, Eqs. (10) and (11) are redundant and the flow evolution is dictated solely by momentum transport.

\section{B. Non-Newtonian flow}

To investigate the non-Newtonian flow regime, the initial temperature is taken to be $675 \mathrm{~K}$ and the pressure gradient $100 \mathrm{MPa} / \mathrm{m}$, which corresponds to a shear loading of $100 \mathrm{MPa}$. The Newtonian viscosity at $700 \mathrm{~K}$ is $\sim 1.2 \times 10^{9}$ Pa s. The steady form of Eq. (9) using the Newtonian viscosity yields a steady-state parabolic profile characterized by $\sim 42 \mathrm{~cm} / \mathrm{s}$ maximum velocity at the center line and $\sim 8.3 \times 10^{-2} \mathrm{~s}^{-1}$ maximum shear rate at the wall. On the other hand, the steady form of Eq. (9) using the nonNewtonian viscosity given by Eq. (15) yields a more localized steady-state profile characterized by $\sim 9 \mathrm{~cm} / \mathrm{s}$ maximum velocity at the center line and $\sim 2.5 \times 10^{-1} \mathrm{~s}^{-1}$ maximum shear rate at the wall. Such non-Newtonian solution however assumes isothermal conditions, i.e., ignores any temperature rise due to adiabatic heating.

The flow evolution computed by the model under these conditions, along with the steady-state Newtonian and nonNewtonian profiles obtained from hydrodynamics, are plot- 


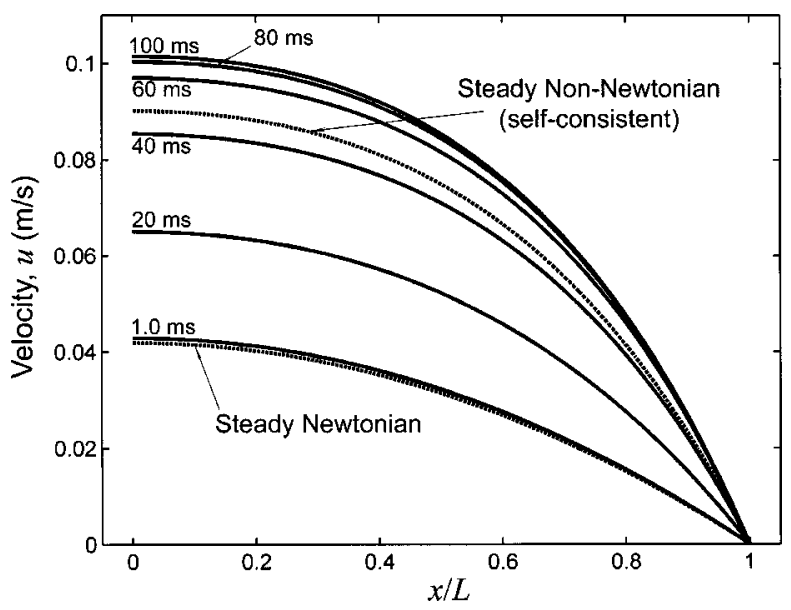

FIG. 6. Evolution of the velocity profile under $675 \mathrm{~K}$ and $100 \mathrm{MPa}$. The velocity gradually departs from its Newtonian limit (lower dotted line) and stabilizes at a non-Newtonian one associated with the flow conditions. The non-Newtonian limit can be evaluated independently (upper dotted line) by considering the shear-rate dependency of flow.

ted in Fig. 6. Instead of relaxing at its Newtonian limit (shown as the lower dotted line in the plot), as would be expected had the flow been Newtonian, the velocity appears to gradually depart from this limit and within $100 \mathrm{~ms}$ to stabilize at another limit near $\sim 10 \mathrm{~cm} / \mathrm{s}$. This higher limit is the non-Newtonian limit associated with the employed flow conditions. It appears that the steady non-Newtonian solution obtained using Eq. (15) is sufficiently close to the evolved profile obtained from solving the complete transport model. The apparent discrepancy can be attributed to a slight increase in temperature $(\sim 0.5 \mathrm{~K})$, which was correctly accounted for in the complete simulation but was ignored in the steady-state modeling. The temperature rise contributed to further viscosity degrading at the wall due to thermal expansion. This result hence suggests that the developed transport model is reliable in modeling non-Newtonian flows since the computed flow evolution appears to be stabilized near a profile that can be obtained independently by assuming structurally static conditions.

In order to further exemplify the system's stabilization to a structural steady state, the evolution of free volume distribution is plotted in Fig. 7. The plot suggests that free volume departs from its equilibrium value $\alpha \nu_{0}\left(T-T_{0}\right)$ and gradually stabilizes at a nonequilibrium linear distribution. This distribution can be obtained independently by means of Eq. (12) as $\alpha \nu_{0}\left(T-T_{0}\right)+R \dot{\gamma} \eta / G$, where $\dot{\gamma} \eta=p^{\prime} x \equiv \sigma$. It can therefore be concluded that under conditions that imply nonNewtonian flow, the model correctly computes the system's structural evolution towards a steady nonequilibrium state.

\section{Shear localization}

To investigate the shear localization flow regime, the initial temperature is taken to be $650 \mathrm{~K}$, while a pressure gradient of $2.0 \mathrm{GPa} / \mathrm{m}$ is assumed corresponding to a shear loading of $2.0 \mathrm{GPa}$. This shear loading is equal to the material shear strength and is the maximum shear loading for which the validity of the model can be sustained.

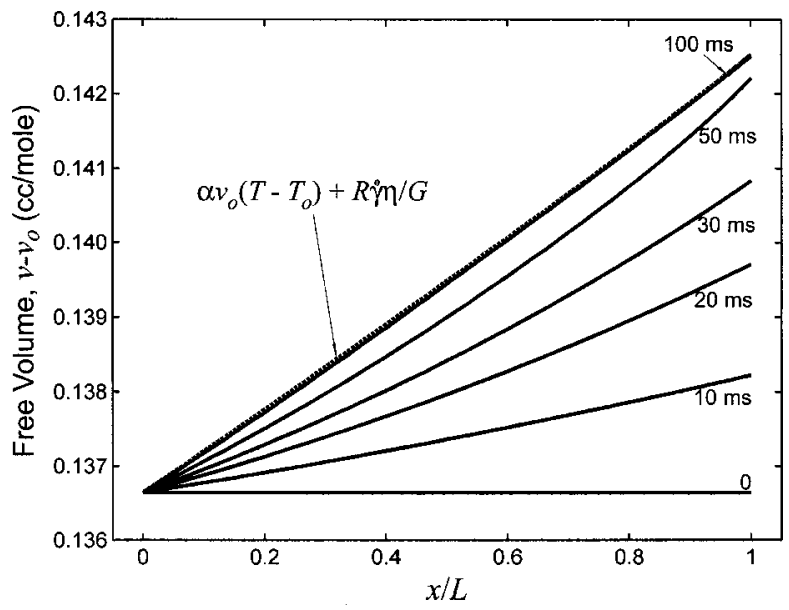

FIG. 7. Evolution of free volume distribution under $675 \mathrm{~K}$ and $100 \mathrm{MPa}$. Free volume departs from its equilibrium value $\alpha \nu_{0}\left(T-T_{0}\right)$ and gradually stabilizes at a linear nonequilibrium distribution given by $\alpha \nu_{0}\left(T-T_{0}\right)$ $+R \dot{\gamma} \eta / G$, as dictated by structurally steady-state conditions.

The system's transient flow response to the induced shear loading is shown in Fig. 8. The plot suggests that the flow departs from its Newtonian limit, however it does not appear to stabilize at another limit but continuously localizes by becoming flatter in the core and steeper near the wall. The flow advancement after localization is concluded, shown in the insert in Fig. 8, suggests that the flow ultimately tends to accelerate unconstrained towards its inviscid (rigid-body translation) limit of $\partial u / \partial t \rightarrow p^{\prime} / \rho$. The transient distribution of shear rate is plotted in Fig. 9. The plot suggests that the shear rate distribution departs from its linear Newtonian value and localizes near the wall, where it gradually approaches a spike-like function tending to blowup. A shear rate distribution that blows up at the point of localization, i.e., a Delta-function distribution, could be perceived as a mathematical representation of a shear band. Hence the shear rate evolution in Fig. 9 could be regarded as the evolution of

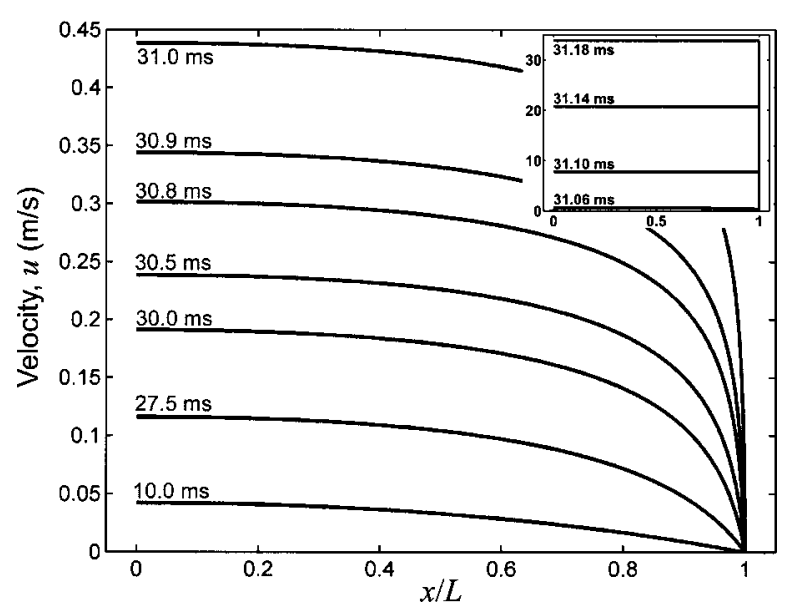

FIG. 8. Evolution of the velocity profile under $650 \mathrm{~K}$ and $2.0 \mathrm{GPa}$. The flow departs from its Newtonian limit and continuously localizes by becoming flatter in the core and steeper near the wall. The flow advancement after localization is concluded (shown in the insert) suggests that the flow ultimately tends to accelerate unconstrained towards its inviscid (rigid-body translation) limit. 


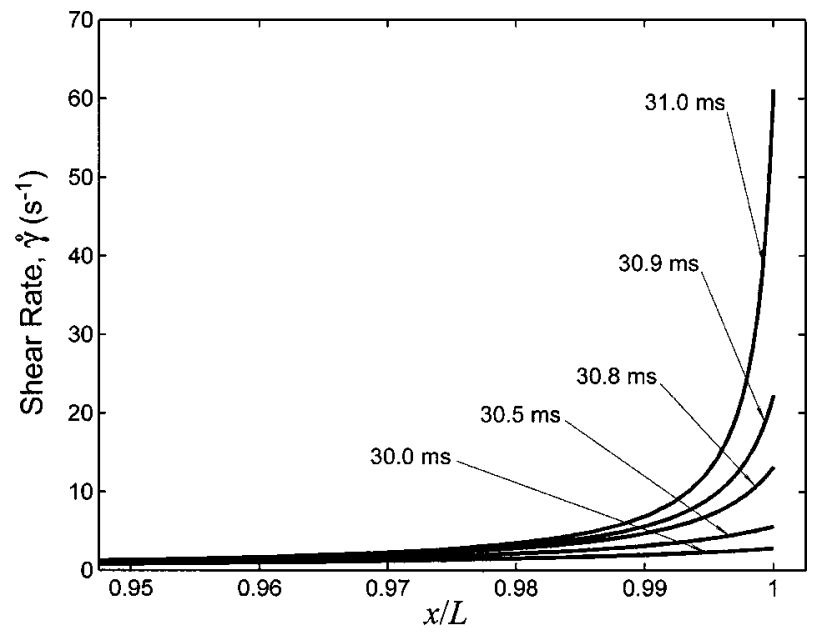

FIG. 9. Evolution of shear rate distribution under $650 \mathrm{~K}$ and $2.0 \mathrm{GPa}$. The shear rate distribution departs from its linear Newtonian value and localizes near the wall, where it gradually approaches a spike-like function tending to blow up. The evolution of shear rate distribution could be regarded as the evolution of a shear band in a bulk liquid during a single serration.

a shear band during a single serration. The evolution of temperature distribution, plotted in Fig. 10, exemplifies the gradual production of energy as a consequence of adiabatic heating. The plot suggests that during the development of shear localization, the temperature rises locally by several degrees. However as localization sets in, the model predicts that temperature rises by several hundreds of degrees approaching the melting point (not shown). This outcome is consistent with experimental observations.

Under the conditions considered here, the shear loading applied to the system is excessively high and contributes to a very high amount of free volume being produced, which fails to annihilate as the relaxation time associated with these conditions is also high. Consequently the system is driven out of structural and thermal equilibrium towards an unstable state characterized by continuous shear localization. Experimental observations on shear band formation suggest that shear lo-

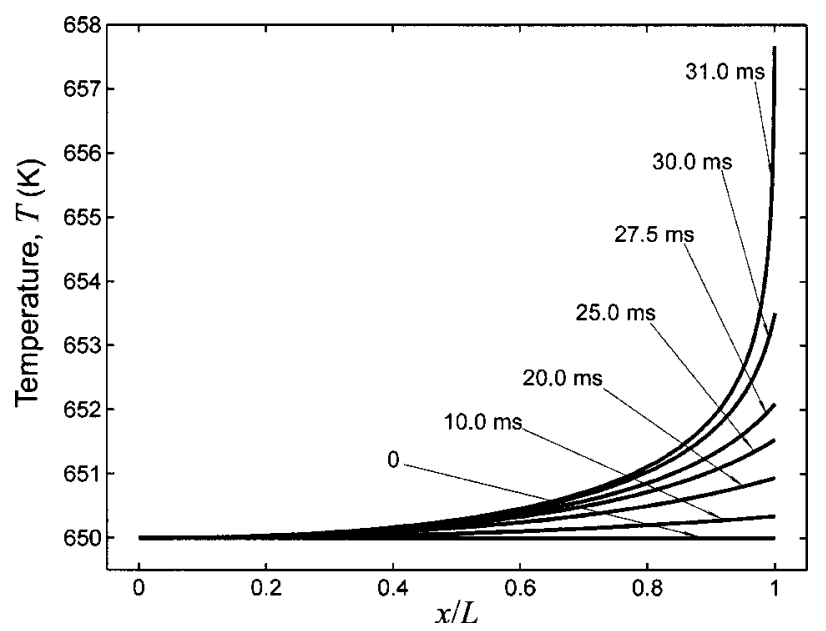

FIG. 10. Evolution of temperature distribution under $650 \mathrm{~K}$ and $2.0 \mathrm{GPa}$. The evolution of temperature distribution exemplifies the gradual production of energy as a consequence of adiabatic heating. During the development of shear localization, the temperature rises locally by several degrees.

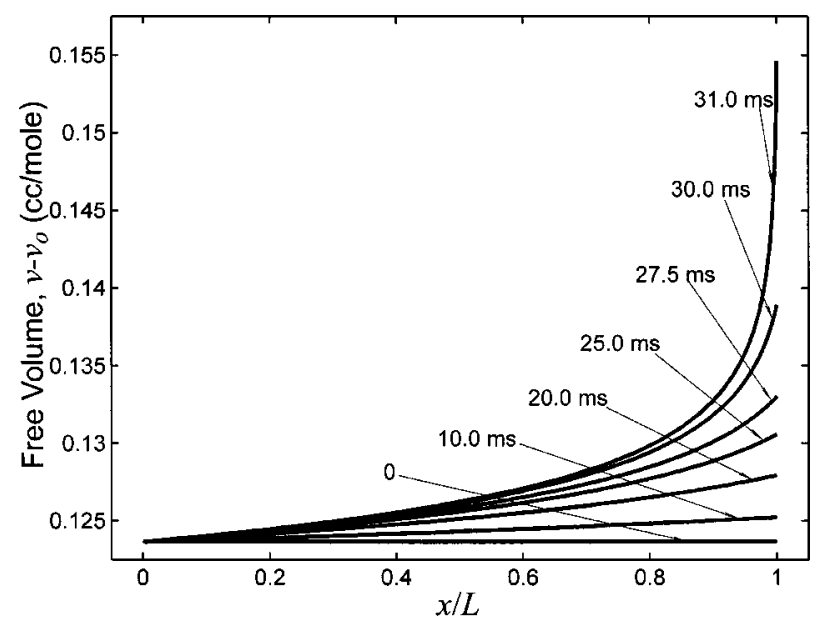

FIG. 11. Evolution of free volume distribution under $650 \mathrm{~K}$ and $2.0 \mathrm{GPa}$. The evolution of free volume distribution exemplifies the gradual production of free volume as a consequence of shearing. Only part of the free volume produced should be attributed to liquid disordering, as significant free volume is generated as a consequence of thermal expansion due to temperature rise.

calization is associated with viscosity degradation, which is a consequence of free volume production. The evolution of free volume distribution, plotted in Fig. 11, exemplifies the gradual production of free volume as a consequence of shearing. It is worth noting that only part of the free volume produced should be attributed to liquid disordering, as significant thermal expansion also takes place as a consequence of adiabatic heating, which causes the equilibrium free volume to increase locally. In order to quantify the deviation from structural equilibrium, the fraction $\left(\nu-\nu_{e}\right) /\left(\nu-\nu_{0}\right)$ is computed locally and its evolution is plotted in Fig. 12. It appears that the system's deviation from structural equilibrium is higher in the vicinity of localization and evolves in time. This suggests that structural nonequilibration could eventually approach $100 \%$ when localization sets in. The evolution of viscosity degradation owing to free-volume pro-

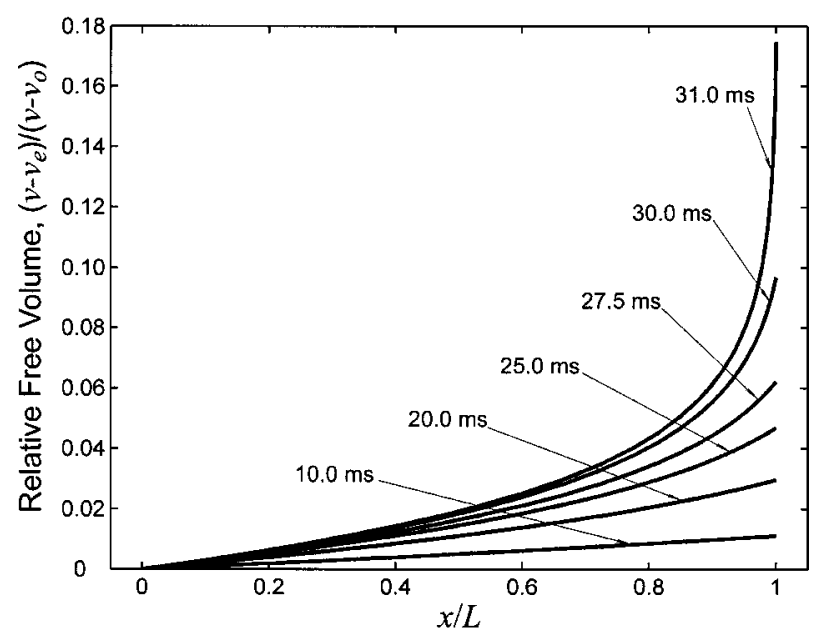

FIG. 12. Fraction of free volume produced by liquid disordering under 650 $\mathrm{K}$ and $2.0 \mathrm{GPa}$. The fraction of generated free volume that can be attributed to disordering is higher in the vicinity of localization and evolves in time, suggesting that it could eventually reach $100 \%$ when localization sets in. 


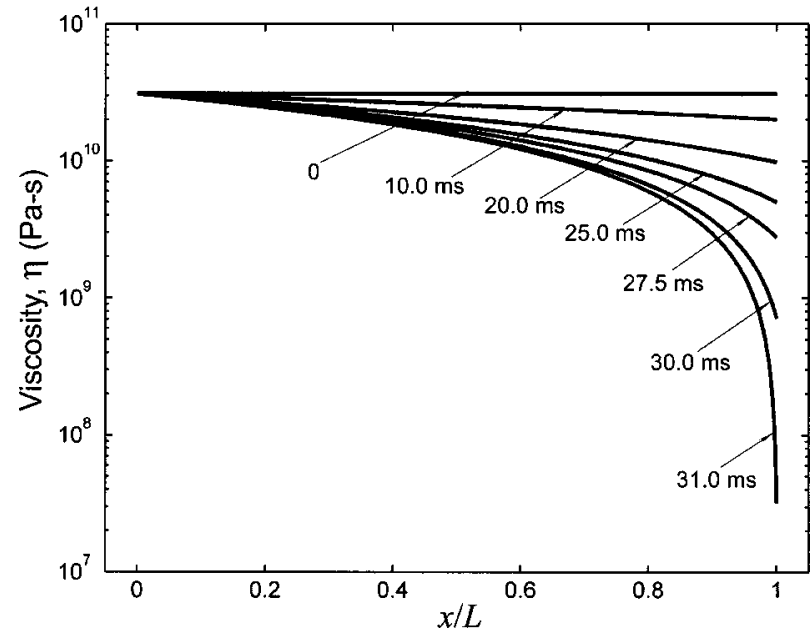

FIG. 13. Evolution of viscosity degradation under $650 \mathrm{~K}$ and $2.0 \mathrm{GPa}$. During a single serration the local viscosity in the vicinity of localization is reduced by 3 orders of magnitude.

duction is shown in Fig. 13. As expected, the plot suggests that during a single serration the local viscosity in the vicinity of localization is reduced dramatically by several orders of magnitude.

\section{DISCUSSION}

The developed transport model appears to successfully simulate the flow characteristics of three distinct flow modes, Newtonian, non-Newtonian, and shear localization. The model is based on a free volume production-rate equation, which comprises free volume creation and annihilation rates whose characteristics are optimized to flow experiments. ${ }^{1,10}$ Therefore, it would be of interest to examine whether the flow simulations yield temperature and shear rate evolutions that could computationally reproduce the experimental flow diagram of Fig. 2.

In Fig. 14 the computed evolution of temperature and

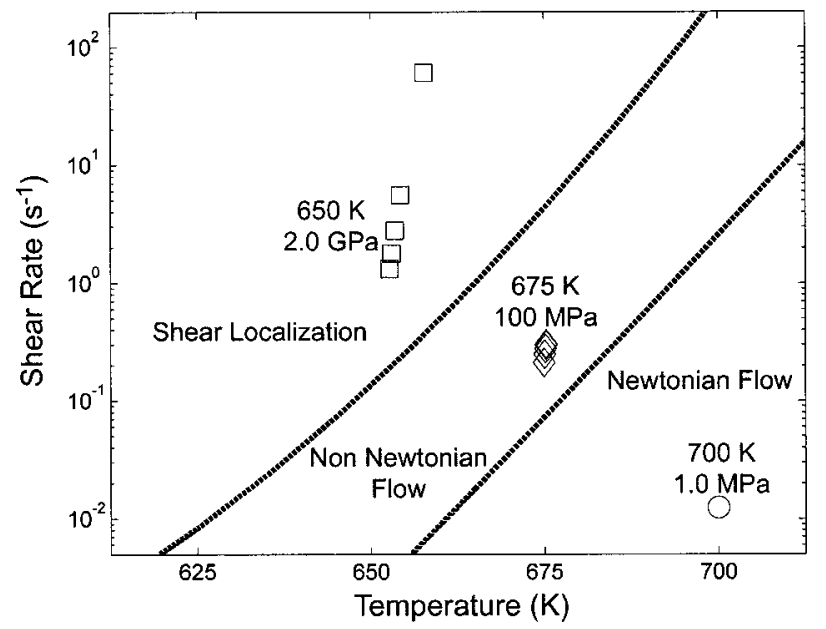

FIG. 14. Computed flow evolutions superimposed on the experimental flow diagram. The temperature and shear rate evolutions computed by the model are bounded within the corresponding experimental flow regimes. This suggests that the model is capable of computationally reproducing the experimental flow diagram. shear rate in the last five time steps for each set of conditions is superimposed on the experimental flow map. In the case of $700 \mathrm{~K}$ and 1.0 MPa the simulation yields static shear rate and temperature responses, i.e., Newtonian flow characteristics, which fall within the experimental Newtonian flow regime. In the case of $675 \mathrm{~K}$ and $100 \mathrm{MPa}$ the simulation yields rather transient shear rate and temperature responses, which eventually stabilize. These are non-Newtonian flow characteristics, and incidentally appear to be bounded within the experimental non-Newtonian flow regime. In the case of 650 $\mathrm{K}$ and $2.0 \mathrm{GPa}$, the simulation yields highly transient shear rate and temperature responses, which appear to become unstable. These are characteristics of shear localization. Although the instability will grow further in time, it appears that the evolution would be bounded within the shear localization regime. Figure 14 therefore suggests that the model is capable to computationally reproduce the experimental flow map.

The simulation results indicate that the nature of flow evolution is essentially dictated by the structural state of the system, which is determined by a balance between free volume creation and free volume annihilation as given by Eq. (4). In order to gain more quantitative insight into the origins of each flow mode, a time scale analysis of Eq. (4) is carried out. To characterize free volume creation, a time scale can be formulated as $\tau_{\nu, \text { cre }} \sim(\dot{\gamma})^{-1} \alpha\left(T_{i}-T_{o}\right) /\left(R / \nu_{o}\right)$. The time scale characterizing free volume relaxation, as used in formulating Eq. (4), is given by $\tau_{\nu, \text { rel }} \sim \eta / G$. Therefore the ratio of this two time scales yields a dimensionless number that could characterize the structural state of the system

$$
\frac{\tau_{\nu, \text { rel }}}{\tau_{\nu, \text { cre }}}=2 \frac{R / \nu_{o}}{\alpha\left(T_{i}-T_{o}\right)} \frac{\sigma}{G} .
$$

A proportionality constant of 2 was incorporated to accomplish consistency with the experimental data. Small values of this dimensionless number would indicate that free volume can be annihilated at a much greater rate than can be created and consequently the liquid would be structurally maintained in stable equilibrium, as Eq. (4) yields $\nu \approx \nu_{e}\left(T_{i}\right)$. Therefore small values would indicate tendency to Newtonian flow. A value near unity suggests that the rates of free volume creation and free volume annihilation are similar, which implies that structurally the liquid would be maintained in a virtual steady state, however it would be far from equilibrium as Eq. (4) yields $\nu \approx \nu_{e}+R \sigma / G$. Hence values near unity would indicate a tendency to non-Newtonian flow. Accordingly, large values of this parameter would indicate that free volume can be created at a much greater rate than it can be annihilated and consequently the liquid would be structurally driven out of equilibrium as well as out of stability, which are characteristics of shear localization. The characteristic numbers that correspond to the sets of conditions employed in this study, which are tabulated in Table I, are in compliance with the above reasoning.

\section{CONCLUSIONS}

A free volume transport equation was formulated by considering a model of local production rate of free volume, 
TABLE I. Values of the dimensionless number characterizing the system's structural state for the conditions considered in the simulation.

\begin{tabular}{lccc}
\hline \hline & $\begin{array}{c}T_{i}=700 \mathrm{~K} \\
\sigma=1.0 \mathrm{MPa}\end{array}$ & $\begin{array}{c}T_{i}=675 \mathrm{~K} \\
\sigma=100 \mathrm{MPa}\end{array}$ & $\begin{array}{c}T_{i}=650 \mathrm{~K} \\
\sigma=2.0 \mathrm{GPa}\end{array}$ \\
\hline$\tau_{\nu, \text { rel }} / \tau_{\nu, \text { cre }}$ & 0.0075 & 0.82 & 18.2 \\
\hline \hline
\end{tabular}

which comprises free volume creation and annihilation rates that are calibrated to experimental flow data in a selfconsistent manner. The free volume transport equation was coupled with momentum and energy transport via viscosity to formulate a system of transport equations to govern the flow of undercooled glass forming liquids. The model was implemented to study the flow behavior of Vitreloy-1 in 1D pressure driven flow.

Experiments on the flow behavior of Vitreloy-1 suggest three distinct modes of flow: Newtonian, non-Newtonian, and localized flow. Each flow mode is associated with a range of shearing rates and temperatures, which extends above glass transition into the undercooled liquid region. The model's capability in simulating the characteristics of each flow mode under different temperature and shear loading conditions is evaluated. For the conditions of $700 \mathrm{~K}$ and 1.0 $\mathrm{MPa}$, the model predicts that the flow profile gradually stabilizes to its Newtonian limit and the liquid is maintained in structural and thermal equilibrium. For the conditions of 675 $\mathrm{K}$ and $100 \mathrm{MPa}$, the model predicts that the flow profile departs from its Newtonian limit and gradually stabilizes to another higher limit, a non-Newtonian one, which is associated with a steady nonequilibrium free volume distribution. The non-Newtonian limit has been evaluated independently by considering the shear-rate dependency of flow, which can be obtained by equating the rates of free volume creation and annihilation, as implied by a structural steady state. For the conditions of $650 \mathrm{~K}$ and $2.0 \mathrm{GPa}$, the model predicts that the flow departs from its Newtonian limit, however it does not stabilize at another limit but continuously localizes and ultimately accelerates unconstrained. Consequently the system is driven out of structural and thermal stability towards an unstable state associated with unconstrained free volume generation, viscosity degradation, and temperature rise.

The temperature and shear rate evolutions computed by the model are superimposed on a temperature-shear rate diagram, and appear to be bounded within the corresponding experimental flow regimes. Therefore it can be concluded that the model is capable to computationally reproduce the experimental flow diagram. The system's structural state that appears to dictate flow behavior is quantified by a dimensionless number, which results from a time scale analysis of the free volume production equation.

\section{ACKNOWLEDGMENTS}

The authors would like to acknowledge the support of the DARPA Defense Sciences Office and the Army Research Office under Grant No. DAAD19-01-1-0525. Valuable discussions with Dr. Sven Bossuyt, Dr. Rainer Birringer, and Theofilos Strinopoulos are also gratefully acknowledged.

${ }^{1}$ J. Lu, G. Ravichandran, and W. L. Johnson, Acta Mater. 51, 3429 (2003).

${ }^{2}$ F. Spaepen, Acta Metall. 25, 407 (1977).

${ }^{3}$ A. I. Taub and F. Spaepen, J. Mater. Sci. 16, 3087 (1981).

${ }^{4}$ P. S. Stief, F. Spaepen, and J. W. Hutchinson, Acta Metall. 30, 447 (1982).

${ }^{5}$ C. A. Volkert and F. Spaepen, Mater. Sci. Eng. 97, 449 (1988).

${ }^{6}$ W. J. Wright, R. Saha, and W. D. Nix, Mater. Trans., JIM 42, 642 (2001).

${ }^{7}$ W. J. Wright, R. B. Schwarz, and W. D. Nix, Mater. Sci. Eng., A 319-321, 229 (2001).

${ }^{8}$ K. M. Flores, D. Suh, R. Howell, P. Asoka-Kumar, P. A. Sterne, and R. H. Dauskardt, Mater. Trans., JIM 42, 619 (2001).

${ }^{9}$ K. M. Flores and R. H. Dauskardt, Acta Mater. 49, 2527 (2001).

${ }^{10}$ W. L. Johnson, J. Lu, and M. D. Demetriou, Intermetallics 10, 1039 (2002).

${ }^{11}$ S. Bossuyt, M. D. Demetriou, W. L. Johnson, and A. L. Greer, Mater. Res. Soc. Symp. Proc. 754, CC7 (2002).

${ }^{12}$ A. Masuhr, T. A. Waniuk, R. Busch, and W. L. Johnson, Phys. Rev. Lett. 82, 2290 (1999).

${ }^{13}$ R. Busch, Y. J. Kim, and W. L. Johnson, J. Appl. Phys. 77, 4039 (1995).

${ }^{14}$ J. Snyder and W. L. Johnson (unpublished data, 1997). 